\title{
A new platform for research and applications with electrons: the PRAE project
}

\author{
Dominique Marchand ${ }^{1 \star}$ on behalf of the PRAE collaboration \\ ${ }^{1}$ Institut de Physique Nucléaire d'Orsay, CNRS/IN2P3 and Université Paris-Sud, UMR8608, Université Paris- \\ Saclay, 91406 Orsay, France
}

\begin{abstract}
The future PRAE multi-disciplinary platform to be built in the campus of the Paris-Sud University (Orsay, France) is based on a high-quality pulsed electron beam in the energy range between 50 to $140 \mathrm{MeV}$. The beam will be delivered to three experimental beam lines, each of them being dedicated to specific research and applications projects related to instrumentation, radiobiology and nuclear physics.
\end{abstract}

\section{Introduction}

The PRAE ${ }^{1}$ project aims at creating in the valley of the University Paris-Sud Campus a multidisciplinary platform dedicated to research and applied physics. The idea of such a platform arose a year ago from the gathering of researchers and engineers from three nearby laboratories, all located in Orsay, namely the laboratory of Imaging and Modeling in Neurobiology and Cancer (IMNC), the Institute of Nuclear Physics of Orsay (IPNO) and the Laboratory of the Linear Accelerator (LAL). The PRAE facility is based on a high performance $50 \mathrm{~Hz}$ pulsed accelerator delivering electrons in the energy range from 50 to $140 \mathrm{MeV}$ to three beamlines, one at a time. The direct beam line, which will benefit from the highest available energy electron beam, will be used to perform radiobiological effect studies to evaluate the potential of an innovative method in radiotherapy based on high energy electrons. A second beam line will be deviated to a fully equipped and instrumented test bench dedicated to detector $R \& D$ and quality checks. The third beam line and its related experimental setup will be devoted to nuclear physics research. The first goal in this field is to exploit the elastic electronproton scattering to determine the proton electric form factor in a very low $\mathrm{Q}^{2}$ range, from a few $10^{-5}$ to $10^{-4}\left(\mathrm{GeV} / \mathrm{c}^{2}\right)^{2}$, providing unique data in an unexplored kinematical domain and contributing to the effort of the communities trying to solve the proton charge radius puzzle.

\section{The electron accelerator}

The PRAE accelerator will consist of a photo-injector, an acceleration section, the beam lines and the related instrumentation. Since a low emittance beam is required, a photo-injector has been chosen as the electron source. Risk minimization guides towards the construction of a gun similar to the one

\footnotetext{
^e-mail: marchand@ipno.in2p3.fr

${ }^{1}$ Platform for Research and Applications with Electrons
} 
constructed for the CLIC "Test Facility 3" at CERN [1] and running successfully for more than four years. The same type of gun is operated at the PHoto-Injector at LAL (PHIL, [2]) platform and will be also used as the injector for the ThomX project now under construction [3] in Orsay. To obtain high charge per bunch, a metallic magnesium photocathode is employed, which can deliver more than $1 \mathrm{nC}$ with a laser pulse energy of a few tens of a $\mu \mathrm{J}$ at a wavelength of $260 \mathrm{~nm}$. The magnesium photocathode could produce the specified charge without requiring an in-situ photo-cathode preparation chamber. The gun is made of 2.5 copper cells, magnetically coupled to a waveguide. To get $1 \mathrm{nC}$ with an emittance lower than $5 \mathrm{~mm} \mathrm{mrad}$, an accelerating field of $80 \mathrm{MV} / \mathrm{m}$ is required, meaning a Radio Frequency (RF) power of $5 \mathrm{MW}$ in a $3 \mu$ s pulse. The electron beam energy at the exit of the gun will be of the order of $5 \mathrm{MeV}$. An industrial laser, synchronized with the RF frequency and triggered at 50 $\mathrm{Hz}$, will be used to extract electrons from the cathode by photoelectric effect. This laser will be installed in an optical room located outside the accelerator concrete shielding and the laser beam will be transported with dielectric mirrors and lenses. The PRAE acceleration section consists of a $3.4 \mathrm{~m}$ long compact accelerator structure based on high-gradient (HG) S-band (3 GHz) cavities. The HG is obtained by means of maximizing the RF efficiency and minimizing the surface fields and the modified Poynting vector at very high accelerating gradients. The HG accelerating structure will be a traveling wave, quasi-constant gradient section and will operate at $3 \mathrm{GHz}\left(30^{\circ} \mathrm{C}\right.$ in vacuum) in the $2 \pi / 3$ mode. The use of the S-band technology allows for a fast implementation enabling the rapid deployment of the first experiments and applications [4]. The final HG structure will provide an energy gain of 65 $\mathrm{MeV}$ for an input peak power of $22 \mathrm{MW}$ with a RF flat top pulse length of $3 \mu \mathrm{s}$ and a repetition rate of $50 \mathrm{~Hz}$. The section will be located after the RF gun as shown in figure 1. After the first project phase

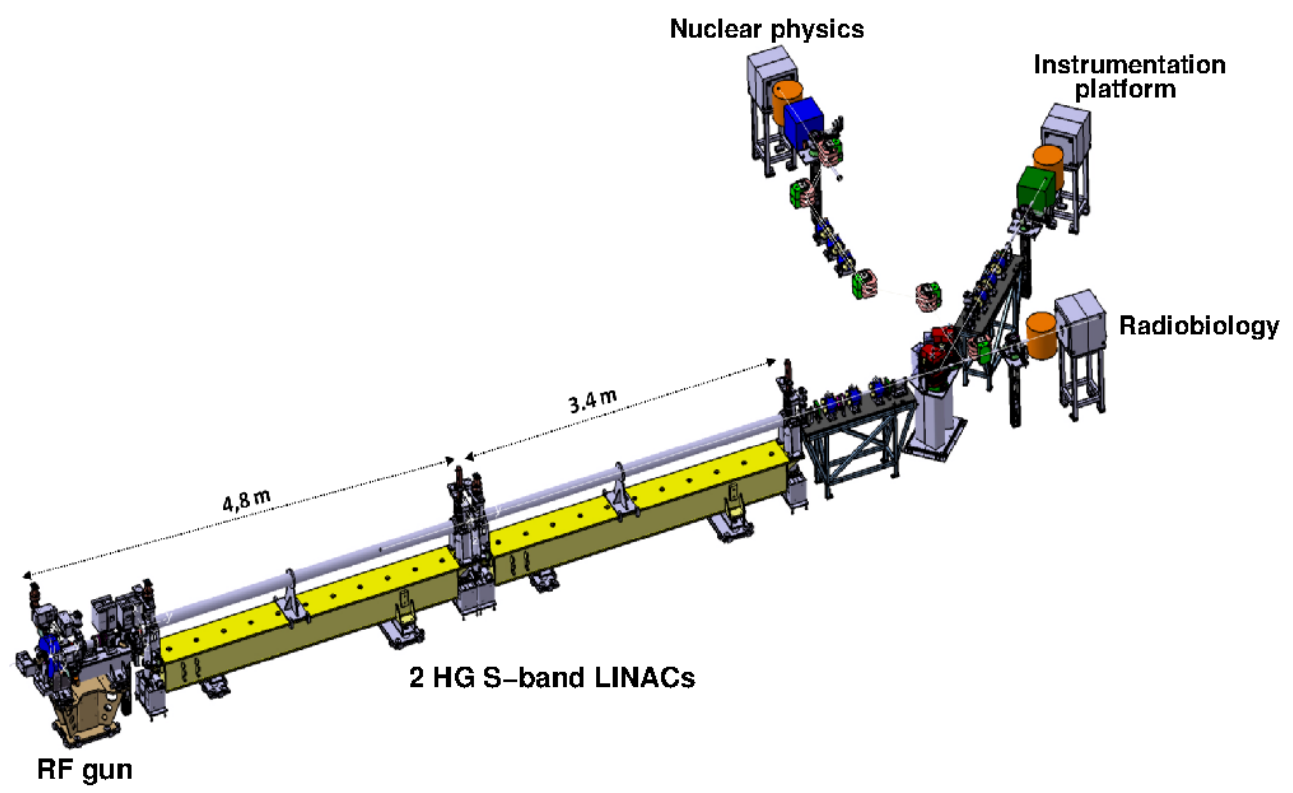

Figure 1. Schematic view of the PRAE general layout corresponding to the second phase of the project (140 MeV electrons). It shows the accelerator structure and the three beam lines dedicated to radiobiology, instrumentation and nuclear physics.

(2017-2019), our goal is to have the accelerator commissioned up to a maximum electron energy of $70 \mathrm{MeV}$ and the three beam lines ready to test their own specific experimental set-up. In a second phase, after 2020, a second high-gradient cavity will be added to reach a $140 \mathrm{MeV}$ electron beam 
energy. The accelerator will be installed in the former site of the LAL linear accelerator benefiting from an existing dedicated infrastructure. The main design parameters of the PRAE accelerator are summarized in table 1.

Table 1. Principle design parameters of the PRAE electron accelerator related to the first project phase $(2018-2019)$ and the second one in brackets $(2020 \rightarrow)$.

\begin{tabular}{|c|c|}
\hline Beam parameters & Project phase 1 (2) \\
\hline \hline Energy range [MeV] & $50-70(100-140)$ \\
\hline Charge (variable) [nC/bunch] & $5 \times 10^{-5}-2$. \\
\hline Normalized emittance [mm.mrad] & $3 .-10$. \\
\hline Radio Frequency [GHz] & 3. \\
\hline Repetition rate [Hz] & 50. \\
\hline Diameter [mm] & 0.5 \\
\hline Bunch length [ps] & $<10$. \\
\hline Energy spread [\%] & $<0.2$ \\
\hline
\end{tabular}

\section{The instrumentation platform}

A fully equipped and instrumented platform will be made available to a user community from the academic and the industrial media willing to test and optimize detectors.

The PRAE facility will provide an adjustable number of particles per pulse in a wide range of multiplicities (current/pulse: $1-10^{10}$ particles) and energies (50-140 MeV). Together with good spatial resolution (beam spot $<1 \mathrm{~mm}$ ) and excellent timing properties (pulse width $<10 \mathrm{ps)}$, this opens a wide range of applications for detector $\mathrm{R} \& \mathrm{D}$. The test bench will be instrumented with a precise beam profile measurement device, a particle counter and a calorimeter. In order to operate remotely the beam diagnostic and detector positioning, we will design and produce a movable chamber at the beam exit. It will comprise a moveable screen for beam position estimate: the operator will observe with the embedded camera the spot produced by the beam on the screen (this technique has been proven at the PHIL/LEETECH facility [2][5]), and adjust the position of the chamber by remotely controlled motors. Then, the screen is removed and the beam profiler is inserted in the beam axis to perform the diagnostic. Once it is performed, the profiler and the screen are removed by a remotely controlled mechanical system. A laser beam for the alignment, fixed on the output of the chamber, will serve to drive the moving support of the detector under test and align it with the beam. In order to perform a fast transverse beam image, a TIMEPIX silicon pixel detector [6] will be made routinely available to the users. It will be integrated in the PRAE acquisition system. The sensors have square pixels with a side of $55 \mu \mathrm{m}$ in a $256 \times 256$ pixels layout covering a square sensitive area of about $2 \mathrm{~cm}^{2}$ and 300 $\mu \mathrm{m}$ thickness. TIMEPIX will be placed in front of the detector under test whenever asked by the user. The number of incident electrons impinging on the detector under tests will be measured by a counter placed just in front of it. This counter is composed of a radiator made of fused silica, where the light emitted by the interacting electrons will be measured by a fast photodetector. A timing resolution of $70 \mathrm{ps}$ for a single electron is expected and the number of electrons will be determined with a precision of 5\%. The setup calorimeter will be realized using BGO scintillator crystals read-out with PMTs or SiPMs. The BGO scintillators provide good stopping power at a relatively low production cost; non-hygroscopicity of the material will allow crystal arrangement in a compact matrix geometry, thus providing an optimal angular coverage and active detection area. The detector will be located along the beamline just behind the detector under test. The latter will be installed at the beam line exit on 
a remotely controlled two-axis trolley with a displacement accuracy of $500 \mu \mathrm{m}$. High voltage power supply channels will be available for customers as well as coaxial $50 \Omega$ cables to get the detector signals, and a multi-purpose data acquisition system (WaveCatcher [7] + NARVAL [8]) serving test detector, sensors, radiation monitors... This common acquisition will ease the event building and the analysis of the detector response under test signals. It has been implemented at LAL for the CORTO [9] test platform, and is currently being implemented at IPNO for the R\&D of the GASPARD detector [10] (in the 64-channel version). The advantage of NARVAL is the definition of a user interface through reusable modules. The work achieved at IPNO, at CSNSM ${ }^{2}$ and at LAL provides templates that will facilitate the installation. In order to test detectors with low intensities, the beamline will be instrumented with a spectrometer that will deliver electrons with adjustable energy from 10 to 140 $\mathrm{MeV}$ at intensities from 1 to 1000 particle(s)/sample. The design of this setup will follow the one of the LEETECH experimental platform that was built at the PHIL accelerator at LAL [2][5].

\section{The radiobiological program}

The motivation for the radiobiological program arose from the fact that whereas radiotherapy is the most frequently used method in cancer treatments [11] it is not fully satisfactory as the dose delivered to a tumor is conditioned by the dose that can be tolerated by the surrounding tissues. In this context, the main challenge in radiotherapy is to find novel approaches allowing the escalation of the dose delivered to the tumor, resulting in an improvement in the cure rate, while lowering the normal tissue complication probability.

In standard radiotherapy (RT), photon beams either X-rays or gamma rays are commonly used. RT with hadrons (protons and light ions) offers several advantages over photons, in particular because they deposit maximum energy at the end of their range, they can be shaped as narrow pencil beams of variable penetration depth and have been shown to be highly effective in treating certain radioresistant tumors thanks to the radiobiological properties of light ions (see e.g. the reviews [12], [13]). However, the cost-effectiveness of Hadron-Therapy (HT) is discussed in literature for some tumor localizations (e.g. prostate cancer), as the construction and running costs of HT units are considerably greater than those of conventional facilities [14].

Electrons are also used in conventional RT, with energies ranging from 5 to $20 \mathrm{MeV}$. However, they are not suitable for the treatment of deep-seated tumors due to their short range and substantial lateral scattering. Since electron beams have a finite effectiveness range after which the dose falls off rapidly, electron beam therapy is typically used in the treatment of superficial tumors. This limitation can be overcome if the electron energy is increased above $70 \mathrm{MeV}$, whereby the penetration depth becomes longer and the transverse penumbra sharper, though the longitudinal penumbra is also increased. Radiation therapy delivered with very high-energy electrons (VHEE) presents an intriguing and possibly cost-effective option to treat cancer. Small diameter VHEE beams can be scanned, therefore, irradiation with VHEE beams requires, in general, less modulation of beam intensities than photon beams [15]. This is very advantageous for intensity-modulated radiation therapy. VHEE beams may be delivered with greater precision and accelerators may be constructed at significantly lower cost than proton beams.

Another approach to increase the therapeutic window for cancer treatment, is to better protect the healthy tissues by using the technique of Spatially Fractionated Radiotherapy (SFR) with submillimetric beam sizes. Such beam sizes allow exploiting the dose-volume effects [16]: the smaller the beam size, the higher the tolerances of the healthy tissue. In SFR, the resulting dose profiles consist of a pattern of peaks and valleys, i.e., with high doses along the beam paths and low doses in

\footnotetext{
${ }^{2}$ Centre de Sciences Nucléaires et de Sciences de la Matière, Orsay, France.
} 
the spaces between them. Such techniques have been proven to be safe and effective in delivering large cumulative doses of radiation in the treatment of cancer. This is due to the fact that areas receiving low (valley) doses serve as re-growth centers for normal tissue, which reduces healthy tissue complications [17]. Remarkably high healthy tissue dose tolerances ( $\geqslant 100 \mathrm{~Gy}$ in one fraction) have been observed in numerous biological experiments using SFR techniques that employ micrometric beams in one direction (see references [18], [19] and [20]), like micro-beam radiation therapy (MRT) or mini-beam radiation therapy (MBRT). MRT and MBRT rendered significant tumor growth delay and, in some cases, complete tumor ablation, see references [18] to [20]. Nevertheless, the biological basis of normal tissue preservation after MBRT irradiations is not well understood yet. In fact, they appear to challenge many of the current paradigms in conventional radiation therapy, since they seem to implicate biological mechanisms different from those involved when direct damage by ionizing radiation takes place. It appears that high Peak-to-Valley Dose Ratio (PVDR) values and low valley doses are requested for healthy tissue sparing [20] while low PVDR values favor tumor control [18]. Considering that point, a possible way of further improving the therapy would be to explore the use of small grid sizes with charged particles, in order to reach very high spatial fractionation of dose in healthy tissue to maximize the sparing, and benefit from the lateral scattering of charged particles to reach a quasi-homogeneous dose in the tumor to increase the tumor control. The objective of the

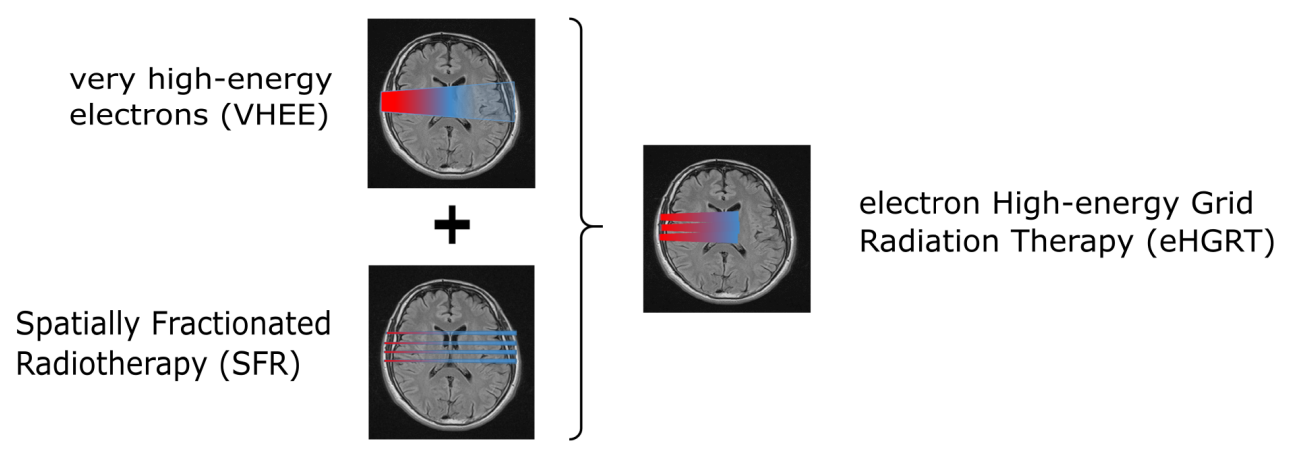

Figure 2. Schematic of the principle of the electron High-energy Grid Radiation Therapy (eHGRT) technique.

radiobiology axis of the PRAE project is to explore such a dose delivery method in electron therapy, combining the use of VHEE beams with the SFR strategy, as illustrated on figure 2. This novel approach will be called: electron High-energy Grid Radiation Therapy (eHGRT) and it is based on a sample or beam scanning used to perform a relative translation of the beam, with respect to the irradiated sample, following a virtual grid as illustrated on figure 3 (left). With this concept, the dose delivered to an hypothetic $8 \mathrm{~cm}$-depth tumor would be quasi-homogeneous while a remarkable tissue sparing is observed at beam entrance, as shown on the figure 3 (right) obtained from simulation with a $150 \mathrm{MeV}$ electron beam. The theoretical proof of concept of this innovative technique has been recently published in reference [21]. In this study, eHGRT has been compared at a dosimetric level with protons grid radiation therapy (pGRT), since the proton-minibeam technique has already been dosimetrically approved in previous study [22]. Both techniques were found suitable (with some configurations of beam size and grid spacing) to treat a $8 \mathrm{~cm}$-depth tumor. The eHGRT shows the highest PVDR in the first centimeters of healthy tissues, improving the tissue sparing before the tumor while pGRT allows more homogeneous dose distribution in the tumor and better tissue sparing behind the tumor. As the dose homogeneity in tumor can be improved by interlacing several beams, these results 

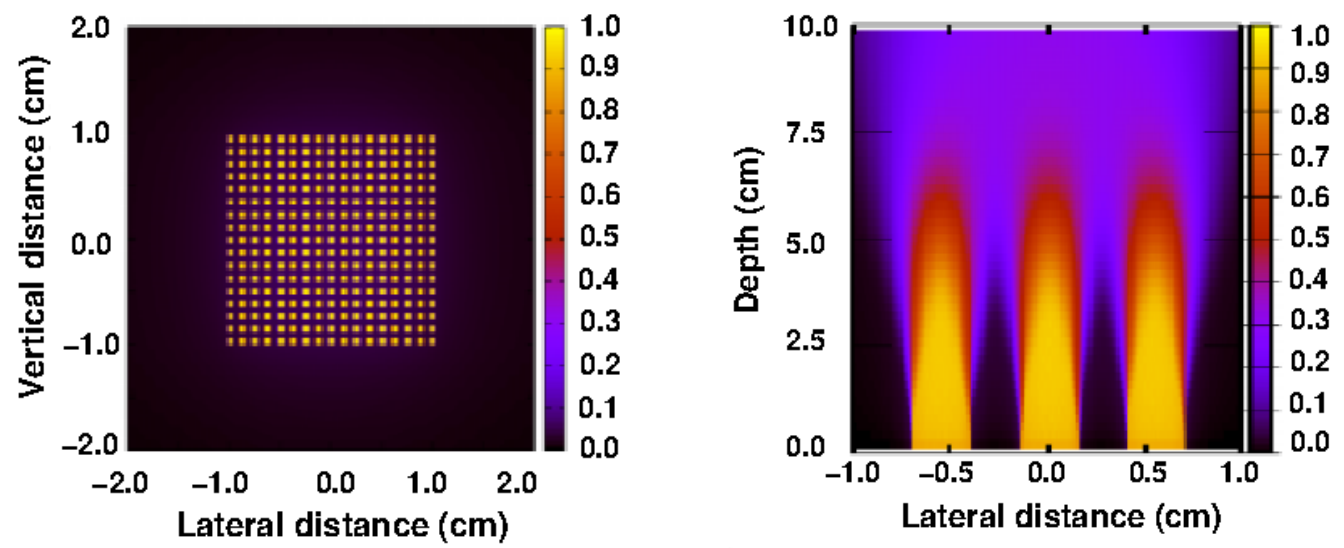

Figure 3. Illustration of the spatial fractionation of the dose with a grid pattern (left side) and the 2D depth-dose distribution in eHGRT (depth versus lateral distance) obtained from [21].

support the interest of performing biological experiments with eHGRT to evaluate this cost-effective grid radiation therapy.

At PRAE, feasibility studies will be performed to accurately evaluate radiobiological effects. A first series of in vivo experiments will also be performed with the main goal to confirm the gain in normal tissue sparing thanks to this novel approach. This program also includes the development of an innovative experimental methodology for a reliable dosimetry with the very small field sizes used, inspired by the dosimetry protocol that was developed for X-rays MBRT [23]. In addition a Monte-Carlo based dose calculation engine will be implemented to guide the pre-clinical trials.

\section{The nuclear physics program}

Our goal is to contribute to the current effort trying to solve the proton charge radius puzzle which arises from the $7 \sigma$ disagreement between the proton charge radius value obtained from the most recent electron scattering experiments $\left(r_{p}=0.879 \pm 0.008 \mathrm{fm} \mathrm{[24])}\right.$ on the one hand, and from Lamb shift measurements with muonic atoms $\left(r_{p}=0.84184 \pm 0.00067 \mathrm{fm}[25]\right)$ on the other hand as illustrated on figure 4 extracted from reference [26].

Several explanations have been proposed [26] to explain this striking discrepancy ranging from inaccurate radius extraction to lepton non-universality, genuine effects and physics beyond Standard Model. As yet no consensus has been reached and as the extrapolation down to $\mathrm{Q}^{2}=0$ is a matter of controversy, the goal of PRAE is to provide high accuracy determination of the proton electric form factor at very low momentum transfer squared from a few $10^{-5}$ up to $10^{-4}\left(\mathrm{GeV} / \mathrm{c}^{2}\right)^{2}$ by measuring the elastic scattering of 50 to $70 \mathrm{MeV}$ electrons off protons with with a $1 \%$ accuracy. In this unexplored very low $\mathrm{Q}^{2}$ range, any deviation from unity of the electric form factor of the proton would indicate the existence of unexpected physics. Besides, the addition of accurate experimental data to existing ones, the $\mathrm{Q}^{2}$ domain considered by PRAE should help to constrain the zero-extrapolation (see references [27], [28] and [29]) required to extract the proton charge radius.

The so-called ProRad (Proton Radius) experiment at PRAE consists of measuring the elastic electron proton scattering cross section over an angular range of the scattered electrons from $5^{\circ}$ to $15^{\circ}$. 


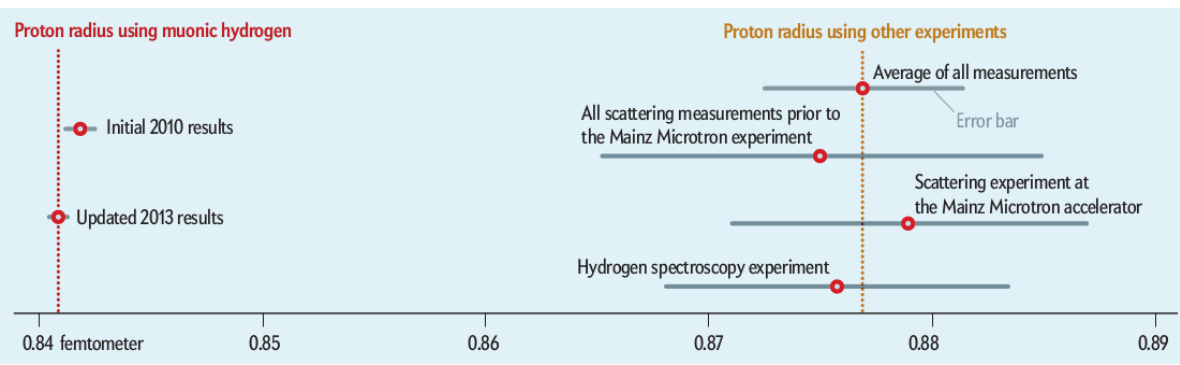

Figure 4. Summary from reference [26] of the proton charge radius values obtained from electron experiments (right) and muon experiments (left).

To select electrons scattered at specific angles we are currently planning to rely on a purely geometrical method avoiding the use of a magnetic field and tracking system. The energy of the electrons is measured in an electromagnetic calorimeter. To define the direction and to get rid of the background a thick shielding with small holes is placed before the detection. Simulations are on-going to define the optimized detection system. Concerning the hydrogen target, different options are considered all based on thin (10 to $20 \mu \mathrm{m}$ ) windowless solid hydrogen targets. Møller scattering electrons, although they represent background to elastic electrons, will be very useful to determine the absolute normalization. As a matter of fact, it is kinematically possible to disentangle Møller from elastic scattered electrons emitted in the same direction by measuring their energy deposition in the same crystal. From figure 5, one can see how much the Møller and the elastic peaks are separated for an electron scattered at $5^{\circ}$ on the left and $10^{\circ}$ on the right for a $50 \mathrm{MeV}$ electron beam energy.
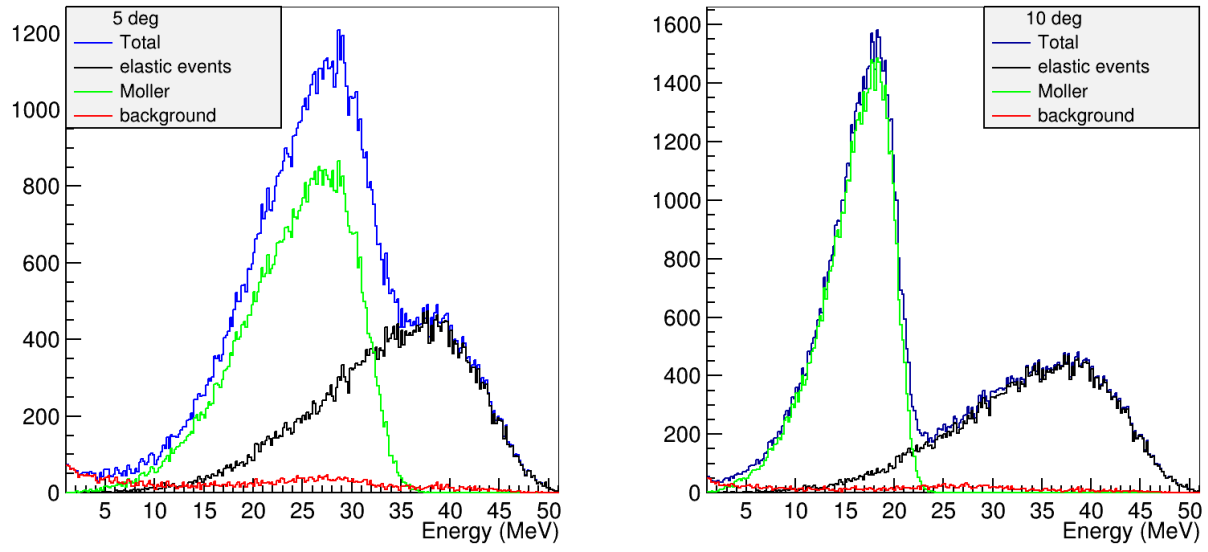

Figure 5. Projection of the total event distribution (blue) in a CSI crystal versus the energy deposit corresponding to $5^{\circ}$ (left) and $10^{\circ}$ (right) for a $50 \mathrm{MeV}$ electron beam energy. The different contributions are: scattered electrons from Møller scattering (green), elastically scattered electrons (black) and background (red). 
Besides the requirements on the position of the beam $\left(\sigma_{x, y}<0.5 \mathrm{~mm}\right)$ and the angular resolution of scattered electrons $(\Delta \theta<1 \mathrm{mrad})$, an accuracy better than $10^{-3}$ is mandatory on the incident beam energy and the radiative effects need to be well evaluated to meet the foreseen sub-percent level on the measurement of the proton electric form factor.

\section{Conclusion}

In a near future, the PRAE multi-disciplinary facility will greatly contribute to the fields of fundamental, as well as applied physics, and will also provide valuable tools for education. Based on a high performance electron accelerator, feasibility studies will be carried out to evaluate the potential of a new innovative method in radiotherapy. A high quality fully instrumented test bench will serve to investigate the performance of detectors and accurate measurements of the proton electric form factor will be performed in an unexplored range of momentum transfer squared from a few $10^{-5}$ down to $10^{-4}\left(\mathrm{GeV} / \mathrm{c}^{2}\right)^{2}$.

\section{Acknowledgement}

This work is partly funded by the CNRS, the French Ile-de-France region in the framework of SESAME projects and the P2IO LabEx (ANR-10-LABX-0038) in the framework « Investissements d'Avenir » (ANR-11-IDEX-0003-01) managed by the French National Research Agency (ANR). Furthermore, we are benefiting from the research conducted in the scope of the IDEATE International Associate Laboratory (LIA) [30].

\section{References}

[1] http://clic-study.web.cern.ch/content/ctf3-general-description

[2] http://http://phil.lal.in2p3.fr/

[3] http://www.lal.in2p3.fr/ThomX

[4] M. El Khaldi, L. Garolfi, in Proc. 6th International Particle Accelerator Conference (Richmond, VA, USA, paper WEPMA008) 2757-2760. ISBN: 978-3-95450-168-7. http://jacow.org/ IPAC2015/papers/wepma008.pdf (2015)

[5] D. Attie et al., e-print: arXiv:1601.04348

[6] X. Llopart, R. Ballabriga, M. Campbell, L. Tlustos, W. Wong, Nucl. Instrum. Methods Phys. Res. A 581, 485-494 (2007). doi:10.1016/j.nima.2007.08.079

[7] D. Breton, E. Delagnes, and J. Maalmi, Nucl. Instrum. Methods Phys. Res. A 695, 61-67 (2012). doi:10.1016/j.nima.2011.12.007

[8] X. Grave, R. Canedo, J.-F. Clavelin, S. Du, E. Legay, 14th IEEE-NPSS Real Time Conference, 119 (2005). doi:10.1109/RTC.2005.1547454

[9] A. Natochii et al., Technical report LAL/RT 15-04 (2015). Retrieved from http://hal.in2p3.fr/ in2p3-01109588

[10] M. Assié et al., The European Physical Journal A 51(1), 11 (2015). doi:10.1140/epja/i201515011-6

[11] E. Niederlaender, KS-NK-06-010-EN-N, Technical report, Eurostat (2006)

[12] R. Orecchia, M. Krengli, B. A. Jereczek-Fossa, S. Franzetti, J. P. Gerard, Critical reviews in oncology/hematology 51(2), 81-90 (2004) 
[13] M. Dosanjh, H. F. Hoffmann, G. Magrin, Nuclear Instruments and Methods in Physics Research Section A: Accelerators, Spectrometers, Detectors and Associated Equipment 571(1), 191-194 (2007)

[14] M. Lodge, M. Pijls-Johannesma, L. Stirk, A. J. Munro, D. De Ruysscher, T. Jefferson, Radiotherapy and Oncology 83(2), 110-122 (2007)

[15] M. Mohiuddin, J. H. Stevens, J. E. Reiff, M. S. Huq, and N. Suntharalingam, Radiat. Oncol. Invest. 4, 41 (1996)

[16] H. J. Curtis, Radiation Research Supplement 7, 250-257 (1967)

[17] A. Dilmanian et al., Proc. Natl. Acad. Sci. U. S. A. 103, 9709 (2006)

[18] P. Regnard et al., Phys. Med. Biol. 53, 861 (2008)

[19] Y. Prezado et al., J. Synchrotron Radiat. 19, 60 (2012)

[20] A. Dilmanian et al., Neuro-Oncol. 4, 26 (2002)

[21] I. Martinez-Rovira and Y. Prezado, Med. Phys. 42, 685 (2015)

[22] C. Peucelle, C. Nauraye, A. Patriarca, E. Hierso, N. Fournier-Bidoz, I. Martínez-Rovira, Y. Prezado, Medical physics 42(12), 7108-7113 (2015)

[23] Y. Prezado et al., Med. Phys. 38, 5012 (2011)

[24] J. C. Bernauer et al., Phys. Rev. C 90, 015206 (2014)

[25] R. Pohl et al., Nature 466, 213 (2010)

[26] J. C. Bernauer, R. Pohl, Scientific American 310, 32 (2014)

[27] K. Griffioen, C. Carlson, S. Maddox, arXiv:nucl-ex:1509.06676

[28] D. W. Higinbotham, A. Amin Kabir, V. Lin, D. Meekins, B. Norum, B. Sawatzky, arXiv:nuclex: 1510.01293

[29] M. O. Distler, T. Walcher, J. C. Bernauer, e-print arXiv:nucl-ex:1511.00479

[30] https://ideate.lal.in2p3.fr/en/home/ 